\title{
$86 \mathrm{GHz}$ VLBI Imaging of the SiO Masers in VX Sgr
}

\author{
S. S. Doeleman \& C. J. Lonsdale \\ Haystack Observatory, Westford, MA, U.S.A.
}

L. J. Greenhill

Harvard-CfA, Cambridge, MA, U.S.A.

\begin{abstract}
We present the first $86 \mathrm{GHz}$ spectral line VLBI map. Strong SiO masers in the late type star VXSgr are mapped with a relative astrometry of 0.15 mas revealing an irregular ring of maser emission that echos structure seen at $43 \mathrm{GHz}$. A surprising feature of this map is the detection of a large $\left(0.5 \mathrm{~km} \mathrm{~s}^{-1} \mathrm{AU}^{-1}\right)$ linear velocity gradient that can be interpreted as rigid rotation of the circumstellar envelope.
\end{abstract}

Circumstellar $\mathrm{SiO}$ masers are often associated with late-type stars, generally Mira variables and supergiants. They occur inside the inner radius of the dust shell, very close to the stellar photospheres, possibly in a region of turbulent outflow (Greenhill et al. 1995). VLBI experiments have demonstrated that the $43 \mathrm{GHz}(\mathrm{v}=1, \mathrm{~J}=1 \rightarrow 0)$ masers lie in ring-like configurations, presumably centered on the star, with tangential rather than radial maser gain paths (Diamond et al. 1994; Kemball \& Diamond 1997).

The SiO maser pump mechanism remains uncertain, and analyses of both collisional pumping (Lockett \& Elitzur 1992) and radiative pumping (Bujarrabal $1994 a, b)$ can be found in the literature. Progress in this area relies on comparing the spatial distribution of different $\mathrm{SiO}$ maser transitions (see for example Miyoshi et al. 1994). The development of $86 \mathrm{GHz}$ VLBI will allow for comparison of the $\mathrm{J}=2 \rightarrow 1$ rotational $\mathrm{SiO}$ masers with the $43 \mathrm{GHz}$ transitions lower on the rotational ladder.

In April 1994 the M-Supergiant VX Sgr was observed as a calibrator during a larger 3mm-VLBI campaign on the Galactic Center. VX Sgr is an M-Supergiant $(\mathrm{D} \sim 1.7 \mathrm{kpc}$ ) whose circumstellar envelope supports strong maser emission in the $\mathrm{SiO} v=1 \mathrm{~J}=2 \rightarrow 1$ transition at $86 \mathrm{GHz}$. Three telescopes participated (Kitt Peak- $12 \mathrm{~m}$, Haystack- $37 \mathrm{~m}$, and OVRO- $4 \times 10 \mathrm{~m}$ ) with no fringes detected on long baselines $(5000 \mathrm{~km})$ to Haystack. The remaining $(u, v)$ coverage on the Kitt Peak-OVRO baseline resulted in an elongated interferometric beam of FWHM $0.4 \times 5.8$ mas with $\mathrm{PA}=32^{\circ}$.

Though $3 \mathrm{~mm}$ spectral line VLBI must contend with small coherence times, the high brightness temperature of the maser spots allowed the use of standard spectral phase referencing techniques as implemented in the NRAO AIPS software package. By referencing the entire map to a bright, point source of maser emission, we achieved astrometry of 0.3 times our beam size.

The resulting $86 \mathrm{GHz}$ image shown here reveals a ring structure of masers around VX Sgr within $\sim 1.5$ stellar radii. This result confirms that the 43 and $86 \mathrm{GHz}$ masers occur in roughly the same region. A two year interval, though, between the $86 \mathrm{GHz}$ map and the last $43 \mathrm{GHz}$ results precludes an exact registration of these transitions. Non detections on long baselines indicate no strong maser emission on scales smaller than $\sim 0.2$ mas. An unexpected finding was the discovery of a large scale linear velocity gradient among the Southern group of masers in VX Sgr. This gradient spans 12 mas ( $22 \mathrm{AU}$ ) with a velocity slope of $0.5 \mathrm{kms}^{-1} \mathrm{AU}^{-1}$. This is the first such linear gradient observed in any SiO 


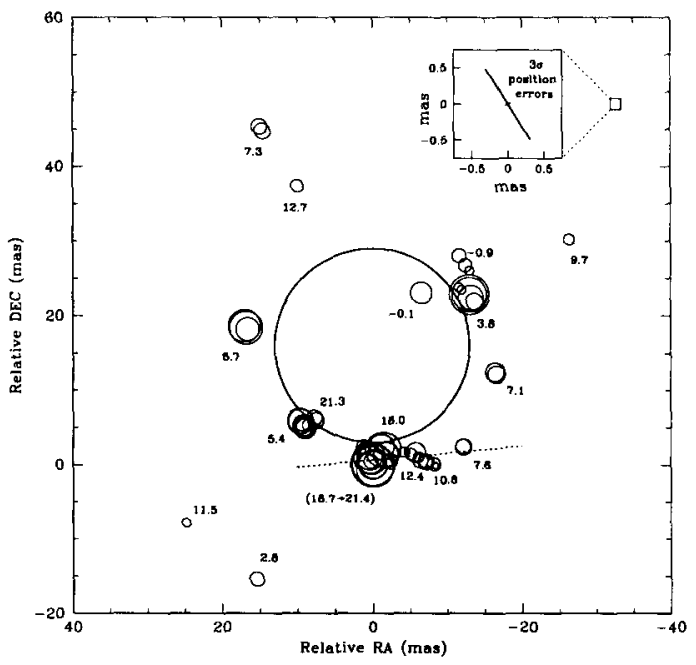

Figure 1. Location and flux density of the brightest $86 \mathrm{GHz}$ maser spots. Each distinct feature is represented by a circle whose radius is proportional to flux density. Flux density ranges from $2 \mathrm{Jy}$ for the weakest component to $14.6 \mathrm{Jy}$ for the phase reference feature which sits at the maps center. Also shown is the disk of VX Sgr with an assumed diameter of $26 \mathrm{mas}$, and a presumed location roughly concentric with the shell of masers. The dotted line shows the direction along which we compute a velocity gradient in the southern complex of maser features.

maser source. If interpreted as rigid rotation, the gradient corresponds to a $v \sin i \sim 13 \mathrm{kms}^{-1}$ with a rotation axis of $\mathrm{PA} \sim 135^{\circ}(\mathrm{E}$ of $\mathrm{N})$. The excellent relative astrometry (tens of $\mu$ as) possible in $3 \mathrm{~mm}$-VLBI maps will allow future inter-transitional tests of co-spatiality on the finest scales. Simultaneous observations at $43 \mathrm{GHz}$ with the VLBA and at $86 \mathrm{GHz}$ with the CMVA are planned.

Acknowledgments. Support for the Coordinated mm-VLBI Array work at the Haystack Observatory is provided under a grant from the National Science Foundation to the Northeast Radio Observatory Corporation.

\section{References}

Bujarrabal, V. 1994a. $A \& A, \mathbf{2 8 5}, 953-970$.

Bujarrabal, V. 1994b. $A \& A, \mathbf{2 8 5}, 971-978$.

Diamond, P. J. et al. 1994. ApJ, 430, L61-64.

Doeleman, S. S., Lonsdale, C. J., \& Greenhill, L. J. 1997. ApJ, submitted.

Greenhill, L. J., et al. 1995, ApJ, 449, 365-375.

Kemball, A. J., \& Diamond, P.J. 1997. ApJ, 481, L111-114.

Lockett, P., \& Elitzur, M. 1992. ApJ, 399, 704-713.

Miyoshi, M. et al. 1994. Nature, 371, 395-397. 